\title{
Analysis on the Symbolic System of Twelve-Phase Tibetan Mask in Baima of Pingwu County
}

\author{
Xinlei Hou, Jingrong Shi \\ College of Urban Planning and Architecture, Southwest Minzu University, Chengdu, China \\ Email: 1483765726@qq.com
}

How to cite this paper: Hou, X.L. and Shi, J.R. (2021) Analysis on the Symbolic System of Twelve-Phase Tibetan Mask in Baima of Pingwu County. Open Access Library Journal, 8: e7592.

https://doi.org/10.4236/oalib.1107592

Received: May 27, 2021

Accepted: June 14, 2021

Published: June 17, 2021

Copyright $\odot 2021$ by author(s) and Open Access Library Inc.

This work is licensed under the Creative Commons Attribution International License (CC BY 4.0).

http://creativecommons.org/licenses/by/4.0/

\section{(c) (i) Open Access}

\begin{abstract}
Twelve phases of Baima Tibetan mask is the national first batch of national intangible cultural heritage, has high research and conservation value, this article from Baima Tibetan and religion, the relation of nature, life, an analysis of the symbolism, twelve-phase mask, to explore the twelve-phase under the mask of cultural symbolic meanings, namely respect religious symbol meaning of the deity, animal worship, hope peace symbol meaning of the human and nature, and yearning for a better life pursuit of symbolic meanings.
\end{abstract}

\section{Subject Areas}

Culture

\section{Keywords}

Baima Tibetan, Twelve-Phase Dance, Twelve-Phase Mask, Symbolism

\section{Introduction}

As the witness and embodiment of the history and culture of the Baima Tibetan, the 12-phase mask has profound connotations and special value. Baima Tibetan is called "east Asia's oldest ethnic group", in the long history, Baima Tibetan people live in relatively closed between mountain, its social structure, the production, and living, religion, national culture is quite unique and original genuine, in Baima Tibetan distinctive national culture, the most representative is "Chigezhou" twelve dancing. Among them, the 12-phase mask in the 12-phase dance has significant characteristics compared with other mask dances, which is of great research value and significance. Starting from the relationship between the Baima Tibetan people and religion, nature, and life, this paper makes a sym- 
bolist analysis of the 12-phase mask, so as to explore the cultural symbolic meaning under the 12-phase mask. At present, academia mainly concentrated on the study of Baima Tibetan ethnic origin, history and culture, religious culture in more macro aspects, mask for less research in the field of micro cultural carrier and so on, in this paper, the study of the twelve-phase mask to explore in order to enrich the study of the microcosmic level of the Baima Tibetan ethnic culture, is the research of Baima Tibetan culture carrier to provide reference and examples, to a certain extent, could also provide the Baima Tibetan ethnic culture protection to certain ideas. This research will find the cultural meaning of Baima Tibetan the 12-phase mask by Symbolic System.

\section{Historical Origin and General Situation of Baima Tibetan in Pingwu County}

The white horse Tibetans are "the oldest ethnic group in east Asia". They are not homologous with the Tibetan, and their ancestors are the Di ethnic group [1]. After a long period of migration, Tibeto-Burman people settled in the mountainous areas of northwest and southwest China, and gradually settled and developed into a powerful Di people. In A.D.383, the former qin defeat at the battle of water, Di regime of the former Qin will then collapse, di today on the corner of Pingwu county, Mianyang city, Sichuan province, aba Tibetan and Qiang autonomous prefecture, Wen county, Longnan city, Gansu province and bureau of Pingwu county in Hangzhong Ningqiang county areas, such as safety considerations for tribes will village location on the mountains, formed a relatively closed tribal structure, the natural economy and the national culture, through long-term development has gradually formed the Baima Tibetan settlements today. In the 1950s, the whole country began the work of ethnic identification and statistics. Due to the high similarity of language and living customs with the Tibetan people and the small number of ethnic groups, the Baima Tibetans were classified as the Tibetan people, and the ethnic name was Baima Tibetans.

Jiuzhaigou Baima Tibetan Village is located in Baima Tibetan Township, Pingwu County, Aba Tibetan, and Qiang Autonomous Prefecture, Sichuan Province, with an area of 715 square kilometers and Duobu River flowing through the whole territory. The township has an average altitude of $2200-2700$ meters, with an annual average temperature of $8-12$ degrees Celsius, a short frost-free period throughout the year, a long sunshine duration, a large temperature difference between day and night, and a low annual accumulated temperature. It belongs to a typical ecological climate zone of high, cold and low temperature. Baima Zang township has a population of 0.2 thousand people. Due to its unique cultural customs, the local tourism develops rapidly and becomes a pillar industry. Baima Tibetan folk culture has bigger differences compared with neighboring nation, the national religion belief for Bon religion and nature, and has its rich and colorful ethnic culture and customs, including 12 with dance and its wear phase mask is the most famous white horse Tibetan and symbolic ethnic 
customs, on May 20, 2005, 12 phase dance upon approval of the State Council of the People's Republic of China on the first batch of state-level non-material cultural heritage list.

\section{Connotation and Characteristics of the Baima Tibetan 12-Phase Mask}

\subsection{Introduction of 12-Phase Mask}

The masks of the Baima Tibetans can be generally divided into three categories: Chi Ge, Chi Mu and Ma Di. Ma Di is the 12-phase mask, and the 12-phase mask also has considerable differences and differences in different regions of the Baima Tibetans. In the Baima Tibetan Township of Pingwu County, the 12phase masks are called "Chou Wu" in the local Tibetan language. They consist of twelve masks, including lion, tiger, leopard, dragon, ox, sheep, quan, feng, diao, big ghost, demon and Chou mo (earth mother), with obvious features of "convex eyes" and "three eyes". [2] twelve-phase mask generally by a prestigious local carpenter is casting, part of the village is by a dedicated crafts heritage of Baima Tibetan twelve-phase mask for manufacturing, according to the history of Baima Tibetan tradition, in the process of manufacturing to be sacred ceremony, so the mask making has more strict processes and specifications. On the selection of materials, the 12-phase mask is wooden, its function is easy to wear and dance, so the general selection of wood for light tung, basswood, etc. in May every year to carry out the collection of wood, after the wood to dry to facilitate carving and the quality of the mask. After the wood is dried, it is carved by carpenters. Carpenters' carving techniques are inherited from their ancestors, and the carving process is a secret period, and the carving techniques should not be disclosed to the public. The carving of the 12-phase mask takes about 10 to 20 days. In local belief, the carving of the 12-phase mask should not be changed at will, otherwise it will be considered to provoke the gods and bring punishment. The 12-phase mask was first colored with the natural plant juice of the mountains and wild areas, and the colors were mainly black, red, yellow and green. After the mask is made, the sacrificial meeting of the Baima Tibetans will carry out a religious ceremony for the mask, which is similar to the Buddhist rites of "opening light" and "summoning the gods".

\subsection{The Application of 12-Phase Mask in 12-Phase Dance}

The twelve-phase dance, also known as dance, is called "Chi Ge Day" in the local Tibetan language, which means "the dance of God". Twelve-phase dance is divided into three categories: Yue, Ganqi and Cuochen, with 42 dance steps. From the sixth day of the first lunar month to the 15th day of the first lunar month, according to the custom of the Baima Tibetans, the ethnic group will set up a blue flag for one day on the wide ground. The Baima Tibetans will wear colorful costumes and 12-phase masks on their heads and begin this special ritual performance. Dance is a sacrificial dance of the Baima Tibetan people. It has the 
beautiful yearning of pleasing the mountain god, exorcising ghosts and avoiding disasters, and praying for blessings. It has high ornamental value and artistic value. By the clan sacrifice, young men and women and the clan in the special dance team for dancing. Baima Tibetans abhor singular numbers, so the number of dancers is even in each twelve-phase dance. Among them, the selection and matching of masks are strictly chosen, so the twelve-phase mask is of high importance. The applications of twelve-phase mask in dancing are as follows:

1) The dancers. Masks of Chi Ge and Chi Mu are of the same gender as those worn by dancers. Zodiac masks are generally unismable in principle. In a specific dance, Chi Ge's mask generally has a threatening appearance, while Chi Mu's mask has a softer line, which shows the characteristics of the mask and the dancer. By the clan sacrifice, young men and women and the clan in the special dance team wearing masks dance.

2) Dance itself. When the wearer of the 12-phase mask dances, the form of the dance is related to the mask. When wearing the lion, tiger, leopard, and other masks, the dancers are required to dance rapidly; when wearing the cow, sheep, ragu, and other masks, the wearer is required to use the steps similar to the farming method such as gentle and crop seeding; when wearing the masks such as big ghost, little devil and "Chou mo", the steps are different.

3) The choice of Mask. Baima Tibetans abhor odd numbers, so the number of dancers is even in each twelve-phase dance. When choosing masks, they usually choose four Chi Ge, Chi Mu and two zodiac masks.

\subsection{The Twelve-Phase Mask and Religion Connection}

The twelve-phase dance is one of the important criteria for the Baima Tibetans to be considered as Tibetans. There are also rich elements of mask dance in the Tibetan religion. Most scholars believe that Bon, the local religion of the Tibetan people before the eighth century $\mathrm{AD}$, has the greatest influence on the Baima Tibetans, which affects the national belief tradition of the Baima Tibetans to some extent. Later in the development, due to the geographical location of Baima Tibetan, more contact with the Tibetan people, Tibetan and Qiang's mask factors also influence the mask of Baima Tibetan culture, some scholars believe that "the elder brother of the pool day" ceremony of change is the concentrated reflection of Baima Tibetan people affected by the Tibetan culture, it also contributed to the 50s in Baima Tibetan ethnic division of work is divided into Tibetan.

\section{Analysis on the Symbolic System of Baima Tibetan Mask}

\subsection{Overview of Symbolism Studies}

Symbolism refers to the expression of rich culture, ideas and spirit through specific and comprehensive images under a certain cultural system. The study of symbolism abroad is relatively comprehensive, which has expanded from literature and anthropology to painting, sculpture, architecture, philosophy, pedagogy 
and other fields. Most domestic studies on symbolism focus on literature and art. Wang Mingning (2020) analyzed symbolism in art creation and argued that symbolism is divided between subjectivity and objectivity in the spatial structure. [3] Xiao-jie yu of symbolism, Belgian novelist George roden Bach's masterpiece "the cloth of He ghost" symbolism analysis that symbolic almost everywhere, different image throughout the course of the full text of a novel real soul. [4] domestic cultural carrier of symbolism research in its infancy, the mask for analysis of the symbolism is less, for a cultural carrier such as mask symbolism analysis can help us better understand the spiritual connotation, is related to the protection of cultural heritage and development play a positive role.

\subsection{Analysis of the Symbolic System of Twelve-Phase Mask}

\section{1) Man and religion}

Twelve phase mask includes the lion, tiger, leopard, dragon, cattle, sheep, chicken, carving, big ghost, imp, "ugly", including eight kinds of animals in the forest, according to the research of the scholars, Baima Tibetan has long insisted that all things have spirit of faith, when this belief with white horse Tibetan habitat for animals, and complex natural environment, presents the obvious characteristics of animal worship [5]. In the long history of development, although their village is relatively closed, the Bama Tibetan people inevitably come into contact with other ethnic groups, especially the Tibetan ancestors, and their national culture is gradually affected by it. It confirms the belief system under the joint influence of Bon religion and national religion, and always firmly believes in the religious belief of animism, which is more concretely reflected in the Twelve Faces Mask. Twelve phase mask in "convex mu", "three eyes" and some elements of Tibetan Buddhism in the qiang's mask, is similar to that of its symbolic meaning to reveal the inviolability of gods and divinity, [6] and more in terms of category, model, color all have many common features. In mask making and mask dancing, there are many ritual procedures presided over by priests to express respect for the gods. At noon or evening, the Baima Tibetans will invite masked dancers to dinner at their homes, where the host will offer special meat as a treat to show respect for the gods and pray for blessings. To sum up, the religious symbolic analysis of the 12-phase mask reflects the symbolic meanings of animal worship, animism and deity of the Baima Tibetan people.

\section{2) Man and nature}

For the Baima Tibetan 12-phase mask, wood is the first material to be selected for the mask, and plant juice is chosen for the paint instead of other materials. In addition to the restriction of the local natural environment, the material selection of the mask also shows a sense of affinity to nature. In addition to the black, red and yellow colors of the earth, fire and sun that are generally expressed by ethnic minorities, green is also added as an important color. In the color expression, green is the most appropriate expression of nature. Based on the classification of the animal character of the 12-phase mask and the animals it shows, it 
can be roughly divided into ferocious type (lion, tiger, leopard, etc.), static type (ox, sheep, raget, etc.) [7].

Among them, the ferocious type is the hunting animal in the deep forest, and the static type is the daily livestock in the life of the Tibetan people in the white horse. Due to the influence of geographical environment, Baima Tibetan has always been a traditional way of farming and hunting with the combination of production, under this kind of mode of production, for the sensitivity of the natural environment is higher, therefore, twelve-phase mask symbol of the complicated attitude towards nature and emotion: both produce attachment to nature, also hope can be in harmony with nature.

\section{3) People and life}

Twelve-phase dance as the Baima Tibetan festival in the grand program, itself is an important part of the Baima Tibetan entertainment and relaxation, the Baima Tibetan in the twelve-phase dance, wearing a fine mask dressed in colorful costumes, singing and dancing happily, no matter how busy the Baima Tibetan working outside, will also return home to celebrate in the festival. This symbolizes the Baima Tibetan people's identity and praise of their national culture, and their pursuit and yearning for a better life.

\section{Conclusion}

In recent years, academic research on the Baima Tibetan people has become hotter and hotter. The 12-phase mask is an important part of the colorful ethnic culture of the Baima Tibetan people, which has high research and cultural value. In this paper, through an analysis of the symbolism, twelve-phase mask, it is concluded that Baima Tibetan. The symbolic meaning of the phase mask, namely respect religious symbol meaning of the deity, animal worship, hope peace symbol meaning of the human and nature, and the yearning for a better life pursuit of meaning, the symbol of the microscopic cultural carrier for Baima Tibetan symbolic analysis and research to provide certain reference and reference.

\section{Funding}

This paper is supported by Southwest Minzu University's Innovative Research Project for Postgraduates in 2021 (No. CX2021SP156).

\section{Conflicts of Interest}

The authors declare no conflicts of interest regarding the publication of this paper.

\section{References}

[1] Xie, X.H. (2004) The Origin of Tibetan Burman People from the Genetic Structure of Chromosome. MSc. Thesis, Fudan University, Shanghai.

[2] Wang, G.J. (2005) The Source of the Three-Eyed-God Mask Worn by the Baima Di Nationality at Dances in Festivals to Exoreise Devils. Journal of Aba Teachers Uni- 
versity, $22,12-13+16$.

[3] Wang, M.N. (2020) The Application of Symbolism in Art Creation. Home Drama, 30, 122-123.

[4] Yu, X.J. (2020) Symbolism in Bruges-la-Morte: A Tentative Analysis. Etudes Francophones, 41, 55-60, 92-93.

[5] Qiao, J. (2015) A Brief Analysis of the Animal Worship Consciousness of "Mask Dance". Home Drama, 25, 160.

[6] Hong, H. (2017) Baima Tibetan Nuo Mask with Qiang's Comparative Study. Journal of Jiamusi University (Social Science Edition), 35, 158-161.

[7] Ma, J. and Chen, J. (2018) Analysis on the "Ferocious Beauty" of Baima Tibetan Tiger Mask. Art Science and Technology, 31, 110-111. 